C-01-2020

\title{
EVALUACIÓN EXPERIMENTAL DE COBERTURAS FLOTANTES MODULARES EN BALSAS DE RIEGO
}

\author{
Martínez-Alvarez, V. ${ }^{(1)}(P)$, Martín-Górriz, B. ${ }^{(1)}$, Maestre-Valero J.F. (1), Gimeno Martínez, \\ J.M. ${ }^{(2)}$, Navarro Fernández F.J. ${ }^{(2)}$
}

\begin{abstract}
${ }^{1}$ Escuela Técnica Superior de Ingeniería Agronómica, Universidad Politécnica de Cartagena. Paseo Alfonso XIII, 48. 30203 Cartagena, España. E-mail: victoriano.martinez@upct.es
\end{abstract}

${ }^{2}$ Arana Water Management S.L. C/Alamo, 23. $1^{\circ} \mathrm{D} .30800$ Lorca (Murcia). E-mail: aranawm@arana-wm.com

\section{Resumen}

Para mejorar la eficiencia global de los sistemas de riego resulta necesario mitigar las pérdidas en balsas mediante técnicas reductoras de la evaporación. Este trabajo contiene los resultados obtenidos tras el ensayo experimental como técnica mitigadora de la evaporación de dos prototipos de cobertura flotante modular, denominados EVAPOCONTROL-2 y EVAPO-CONTROL-G4. Los prototipos fueron evaluados durante sendos años en balsas agrícolas del sureste español con el fin de (1) determinar experimentalmente su eficiencia como tecnología para la reducción de la evaporación, y (2) verificar experimentalmente su efecto sobre la calidad de agua almacenada para su aplicación en riego agrícola.

La cobertura EVAPO-CONTROL-G4 ha conseguido reducir la evaporación en un $77,52 \%$ durante el ensayo experimental de un año en Puerto Lumbreras, mejorando ligeramente los resultados del prototipo anterior EVAPO-CONTROL-2, que redujo la evaporación en un $76,16 \%$ al ser evaluado durante un año en una balsa experimental localizada en el Campo de Cartagena. Por tanto, este ensayo confirma que se trata de una tecnología eficiente para la reducción de la evaporación en balsas de riego, y que el nuevo prototipo mejora el comportamiento de la versión anterior. El factor de reducción de la evaporación no ha sido constante a lo largo del año, sino que ha variado desde el $70,80 \%$ en el mes de enero hasta el $81,56 \%$ en el mes de agosto.

Respecto a los efectos de las coberturas EVAPO-CONTROL-2 y EVAPO-CONTROLG4 sobre la calidad del agua, la conductividad eléctrica se ha mantenido en valores muy reducidos y condicionados a la entrada de agua directa de lluvia. La concentración de clorofila-a (proxy de presencia de algas en la balsa) y el valor de turbidez también ha sido prácticamente nulos tras la instalación de la cobertura flotante, evidenciando la eficiencia de la cobertura como técnica de control de la concentración de microalgas en la balsa de riego.

\section{1- Introducción}

En las regiones áridas y semiáridas, donde los recursos hídricos suelen ser deficitarios e irregulares, las balsas de riego se han convertido en un elemento imprescindible para la aplicación de sistemas de riego de alta frecuencia. Con la modernización de regadíos, se ha producido un desarrollo generalizado de estas infraestructuras de almacenamiento y regulación de agua. La proliferación de las balsas ha repercutido en la eficiencia global de los sistemas de distribución de riego como 
consecuencia de las importantes pérdidas de agua por evaporación que se producen en las mismas. En este sentido, sólo en la cuenca del Segura Maestre Valero et al. (2013) estimaron unas pérdidas de 66,94 hm3/año en balsas de riego, frente a 23,67 hm3/año en grandes embalses.

Para mejorar la eficiencia global de los sistemas de riego resulta necesario mitigar las pérdidas en balsas mediante técnicas reductoras de la evaporación. Son numerosas las técnicas que se han experimentado con el fin de reducir la evaporación en masas de agua, entre las que se encuentran la aplicación de sustancias químicas poco volátiles (monolayers), la implantación de coberturas flotantes o suspendidas, la desestratificación del cuerpo de agua, los cortavientos vegetales, etc. (Martínez et al., 2006; Craig et al., 2005; Brown, 1988). Gran parte de las mismas resultan poco atractivas para su empleo en balsas de riego como consecuencia de su baja eficiencia, de su elevado coste, de la incompatibilidad con las características específicas de las balsas y del uso posterior del agua, de la dificultad de instalación, etc.

En los últimos años las coberturas flotantes modulares de bajo coste se han convertido en una alternativa atractiva para el sector del regadío, ya que sus costes son competitivos en comparación con los de otras técnicas y no requieren obras o actividades específicas para su instalación, ni tampoco tareas de mantenimiento a lo largo de su vida útil.

\section{2- Objetivos}

Este trabajo contiene los resultados obtenidos tras el ensayo experimental de dos prototipos de cobertura flotante modular, denominados EVAPO-CONTROL-2 y EVAPOCONTROL-G4. Los prototipos fueron evaluados durante sendos años en balsas agrícolas del sureste español con el fin de (1) determinar experimentalmente su eficiencia como tecnología para la reducción de la evaporación, y (2) verificar experimentalmente su efecto sobre la calidad de agua almacenada para su aplicación en riego agrícola.

\section{3- Materiales y Métodos}

La caracterización técnica de las coberturas se ha basado en la monitorización y análisis de datos de evaporación y calidad de agua durante un periodo de 12 meses. La cobertura EVAPO-CONTROL-2 se evaluó del 19 de febrero de 2018 al 18 de febrero de 2019) en una balsa de riego ubicada en Cartagena, de $1500 \mathrm{~m}^{2}$ de superficie y capacidad máxima de $6700 \mathrm{~m}^{3}$. La cobertura EVAPO-CONTROL-G4 se evaluó del 11 de enero de 2019 al 10 de enero de 2020 en una balsa de riego ubicada en Puerto Lumbreras, de $600 \mathrm{~m}^{2}$ de superficie aproximada y capacidad máxima de $2000 \mathrm{~m}^{3}$.

Para evaluar la eficiencia de la coberturas como técnica reductora de la evaporación se han comparado las medidas de evaporación en la balsa cubierta $\left(E_{c}\right)$, registradas mediante un transductor de presión de alta precisión sumergido, con los correspondientes a la balsa descubierta $(E)$, estimados con un modelo de evaporación en masas de agua desarrollado por el equipo investigador (Martínez Alvarez et al., 2007). Para la aplicación del modelo (hipotética balsa descubierta) se han utilizado registros diarios de las siguientes variables meteorológicas en las inmediaciones de la balsa: radiación solar global, precipitación, velocidad del viento, temperatura y humedad relativa del aire.

Para evaluar el efecto de las coberturas sobre la calidad de agua almacenada se han registrado con una frecuencia mensual los siguientes parámetros físico-químicos a lo largo 
del perfil del agua con una sonda multiparamétrica OTT DS5: temperatura, $\mathrm{pH}$, conductividad eléctrica, oxígeno disuelto, turbidez y clorofila-a (usado como proxy de la cantidad de algas).

A continuación se detallan distintos aspectos metodológicos sobre (1) el sistema de adquisición de datos y los sensores instalados, (2) la estimación de la evaporación en la balsa descubierta mediante la modelización de su balance de energía, (3) los datos meteorológicos empleados en la modelización de la balsa descubierta, y (4) la adquisición de datos de calidad de agua.

\section{1- Adquisición de datos y sensores instalados}

La Fig. 1 presenta el diseño experimental:

- La tasa de evaporación en el embalse cubierto $\left(E_{c}\right)$ se determinó a partir de medidas de nivel de agua registradas con un sensor de presión (Druck, PDCR1830, precisión $\pm 0,06 \%$ en una escala de $75 \mathrm{mbar}$ ). El sensor se situó en el interior del embalse unido a un lastre para evitar su movimiento.

- La evolución del perfil térmico del embalse se registró mediante 6 sensores de temperatura (T-107, Campbell $\mathrm{SCl}$ ) sumergidos en el cuerpo de agua, que se anclaron a una cadena suspendida desde el lateral de la balsa.

- La velocidad del viento en la balsa se registró con un anemómetro de cazoletas (A100R, Campbell SCl) situado a $2 \mathrm{~m}$ de altura junto a la balsa, con el fin de tener registros de la velocidad del viento más representativos de las condiciones locales que los registrados en las estaciones del SIAM CA-12 (Cartagena-La Palma) para el prototipo EVAPO-CONTROL-2 y LO-61 (Puerto Lumbreras-El Esparragal) para el prototipo EVAPO-CONTROL-G4.

- Sensor de condensación para detectar la presencia de condensación atmosférica sobre la cobertura (S237, Campbell SCI).

- Datalogger Campbell CR1000 para el almacenamiento de los datos registrados. Los datos mencionados se registraron automáticamente cada $10 \mathrm{~s}$, además de obtener los valores medios horarios y diarios.

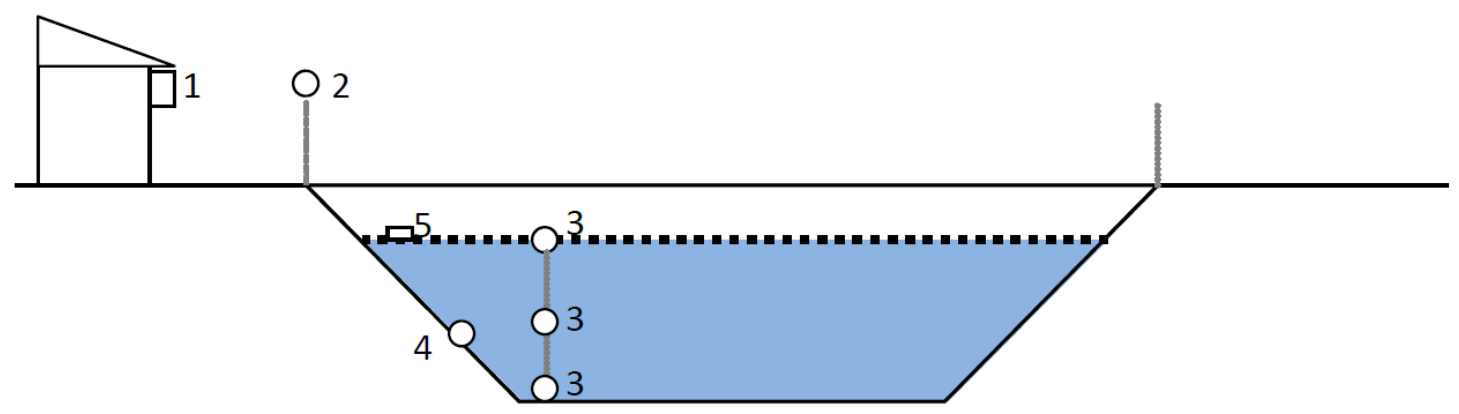

Figura 1. Esquema del embalse experimental con (1) datalogger, (2) anemómetro, (3) sensores de temperatura del agua, (4) sensor de presión y (5) sensores de presencia de condensación.

\section{2- Modelización de la evaporación en la balsa descubierta}


El balance de energía de un cuerpo de agua se determina como las ganancias menos las pérdidas de calor en un intervalo de tiempo determinado. Basado en las principales leyes físicas de la conservación de la energía, el balance de energía puede expresarse del siguiente modo:

$$
R_{n}+\lambda E+H_{s}+Q_{w}=0
$$

donde $R_{n}$ es la radiación neta en la superficie, $\lambda E$ es el flujo evaporativo, $H_{s}$ es el flujo de calor sensible intercambiado con la atmósfera y $Q_{w}$ es la variación de calor almacenado durante el intervalo de tiempo considerado. Todos los flujos están expresados en $\mathrm{Wm}^{-2}$, $E$ es la tasa de evaporación $\left(\mathrm{kg} \mathrm{m}^{-2} \mathrm{~s}^{-1}\right)$ y $\lambda$ el calor latente de vaporización $\left(\mathrm{J} \mathrm{kg}^{-1}\right)$. El criterio de signos es el siguiente: todos los flujos positivos indican aporte de calor a la superficie (por tanto energía disponible para el proceso de evaporación) y los negativos corresponden a las pérdidas de calor de la superficie. A continuación se detalla el procedimiento de cálculo de cada uno de los términos del balance para la balsa en estudio.

La radiación neta es la resultante el balance de radiación de onda corta y larga recibida y perdida por la superficie de agua:

$$
R_{n}=R_{s}-\alpha R_{s}+R_{a}-R_{w}
$$

donde $R_{s}$ es la radiación solar incidente (onda corta), $\alpha$ el albedo (porcentaje de $R_{s}$ reflejado por la superficie), $R_{a}$ la radiación atmosférica (onda larga) y $R_{w}$ el calor emitido por la superficie en función de su temperatura (onda larga). El valor medio anual de $\alpha$ para embalses en la latitud correspondiente al sur de España puede considerarse igual a 0,06. Los términos de onda larga del balance se han obtenido del siguiente modo: $R_{a}$ se ha determinado con la metodología propuesta por la FAO (Allen et al., 1998), a partir de las variables meteorológicas $T_{a}, H R$ y $R_{s}$ registradas en la estación, mientras que $R_{w}$ se ha calculado con la ecuación de Stefan-Boltzman, considerando que el valor de la emisividad del agua es 0,97 .

La tasa de evaporación de la balsa descubierta $\left(E, \mathrm{~mm}^{\text {día }}{ }^{-1}\right)$ se ha calculado por medio de la siguiente ecuación de transferencia de masa:

$$
E=h_{v}\left(e_{s}-e_{a}\right)
$$

donde $e_{s}$ y $e_{a}$ son la presión de vapor de saturación a la temperatura de la superficie del agua, $T_{w}$, y la presión de vapor actual a la temperatura del aire, $T_{\mathrm{a}}$, respectivamente, y $h_{v}$ es el coeficiente de transporte de vapor de agua. Generalmente se asume que $h_{v}$ es proporcional a la velocidad del viento a $2 \mathrm{~m}$ de altura y dependiente del área de la superficie evaporante $\left(S, \mathrm{~m}^{2}\right)$ a través de una función empírica $f(S)$. En la bibliografía pueden encontrarse diferentes expresiones de cálculo de $f(S)$, entre las cuales se ha seleccionado para este estudio la propuesta por Martínez et al. (2007), que a su vez combina las funciones empíricas propuestas por Molina et al. (2004), Brutsaert (1982) y Harbeck (1962), siendo válida para el rango de superficie: $1 \mathrm{~m}^{2}<S<10^{10} \mathrm{~m}^{2}$.

El calor sensible intercambiado en la interfase aire-agua viene dado por:

$$
H_{s}=h_{c} \rho C_{p}\left(T_{a}-T_{w}\right)
$$

donde $C_{p}$ es el calor específico del aire $\left(\mathrm{J} \mathrm{kg}^{-1} \mathrm{~K}^{-1}\right), \rho$ es la densidad del aire $\left(\mathrm{kg} \mathrm{m}^{-3}\right), T_{a}$ y $T_{w}$ son la temperatura del aire y de la superficie de agua, respectivamente, y $h_{c}$ es el coeficiente de transferencia por convección agua-superficie. El coeficiente $h_{c}$ puede aproximarse al valor del coeficiente empírico de transporte de vapor de agua, hc (Analogía de Reynolds), utilizado en el cálculo de la tasa de evaporación:

$$
h_{c}=\lambda \text { Y } h_{v}
$$

donde $y$ es la constante psicrométrica $\left(\mathrm{kPa} \mathrm{K}^{-1}\right)$. 

ecuación:

El calor almacenado o cedido por el cuerpo de agua viene dado por la siguiente

$$
Q_{w}=C_{w} P \Delta T_{w} / \Delta t
$$

donde $C_{w}\left(\mathrm{~J} \mathrm{~kg}^{-1} \mathrm{~m}^{-3}\right)$ es la capacidad calorífica volumétrica del agua, función de su temperatura, $P(\mathrm{~m})$ es el espesor o profundidad del cuerpo de agua, y $\Delta T_{w} / \Delta t$ es la variación de la temperatura del agua durante el intervalo tiempo considerado. $P$ se determina a escala diaria en función del área y volumen de la balsa.

Todos los términos del balance de energía dependen de la temperatura de la superficie de la superficie. El algoritmo de cálculo empleado, consiste en la realización de iteraciones hasta encontrar la temperatura objetivo para la cual se cumple la condición de equilibrio. El modelo ha sido validado con datos experimentales para el cálculo de la evaporación en balsas de riego (Gallego et al., 2009).

\section{4- Resultados}

La cobertura EVAPO-CONTROL-2 ha conseguido reducir la evaporación en un $76,16 \%$ durante el ensayo experimental de un año, por lo que se confirma como una tecnología eficiente para la reducción de la evaporación en balsas de riego. El factor de reducción de la evaporación no ha sido constante a lo largo del año, sino que ha variado desde el $70,5 \%$ en el mes diciembre hasta el $79,4 \%$ en los meses de agosto y septiembre. Esta variación del factor de reducción de la evaporación mensual ha mostrado una clara correlación lineal $\left(R^{2}=0,89\right)$ con la temperatura del aire media mensual. La Fig. 2 recoge la evolución de la tasa de evaporación en la balsa experimental medida durante el ensayo $\left(E_{c}\right)$ y la tasa de evaporación modelizada para el embalse descubierto $(E)$, habiéndose eliminado los 37 días en los que ha habido precipitaciones significativas o alguna incidencia en la adquisición de datos.

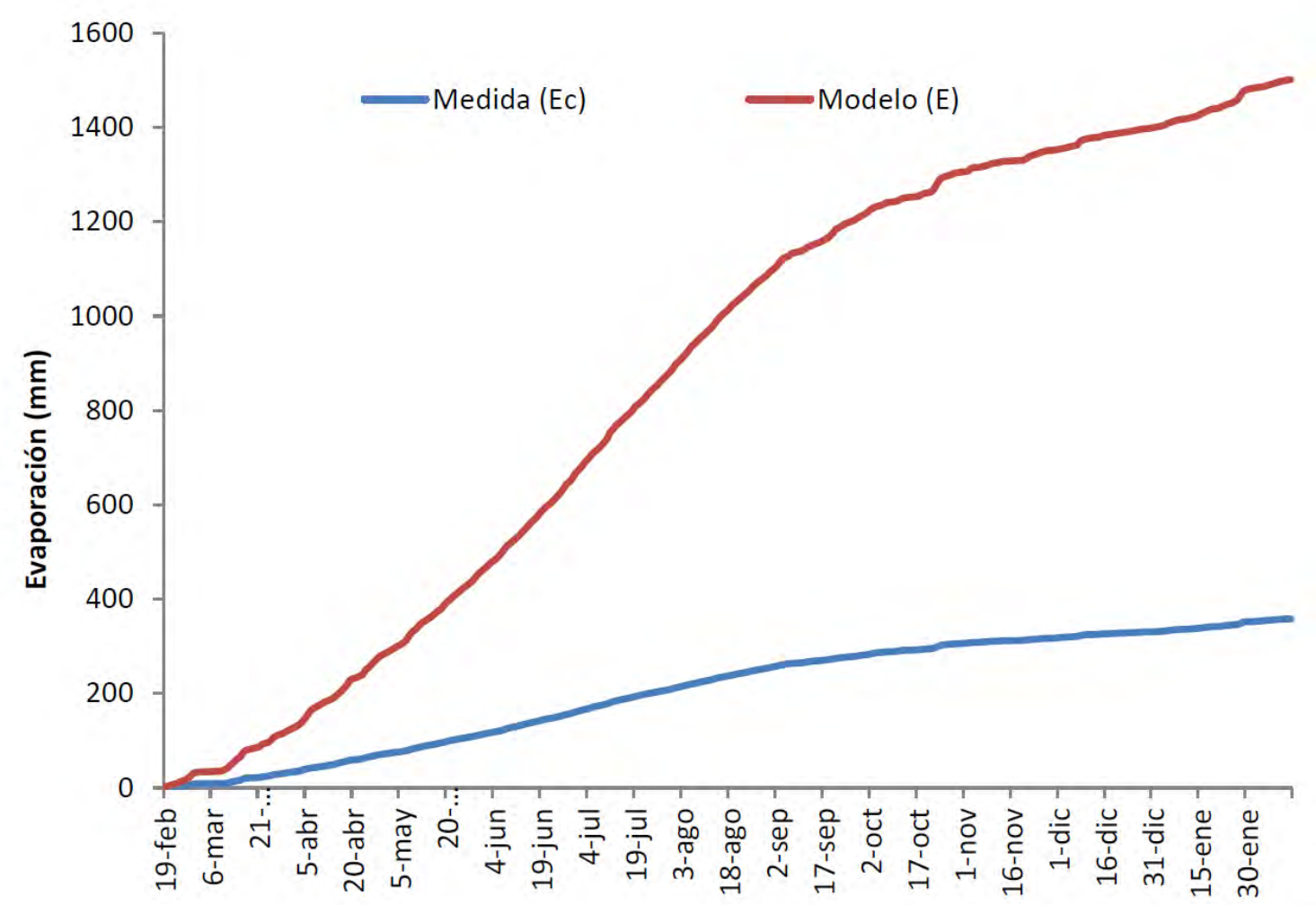

Figura 2. Evolución de la tasa de evaporación de la cobertura EVAPO-CONTROL-2 durante el ensayo. 
La cobertura EVAPO-CONTROL-G4 redujo la evaporación en un 77,52\% durante el ensayo, mejorando ligeramente los resultados del prototipo anterior EVAPO-CONTROL-2. EI factor de reducción de la evaporación ha variado desde el $70,8 \%$ en el mes de enero hasta el $81,5 \%$ en el mes de agosto. Además, el nuevo prototipo mejora el comportamiento mecánico de la versión anterior, en el que el valor umbral de velocidad de viento en el que se producía el giro de los módulos se encontraba en torno a los $15 \mathrm{~km} / \mathrm{h}$. Con el prototipo EVAPO-CONTROL-G4 no se ha observado este fenómeno durante el ensayo, circunstancia que puede justificar su mejor comportamiento como técnica reductora de la evaporación. La Fig. 3 recoge la evolución de se tasa de evaporación en la balsa experimental medida durante el ensayo.

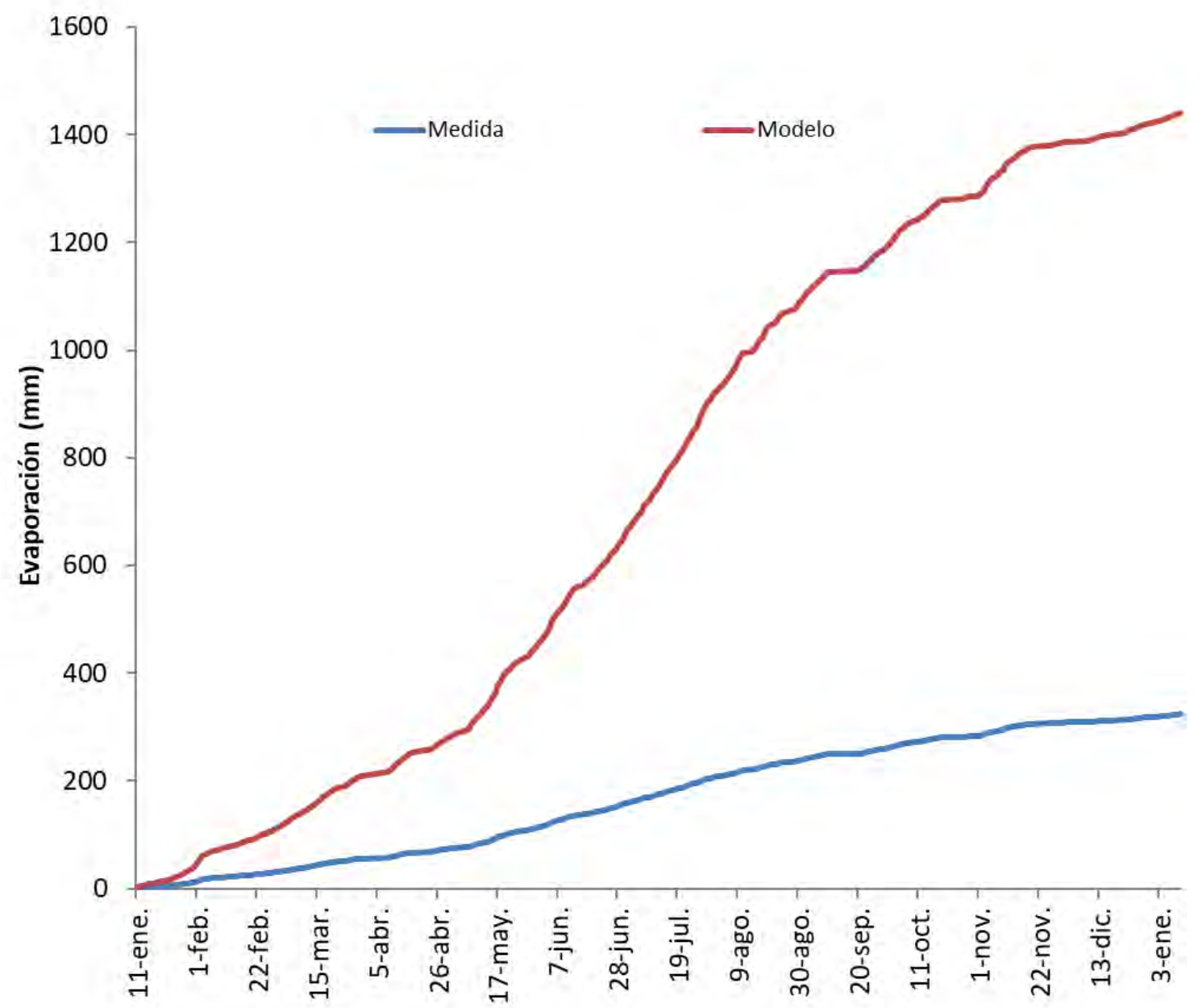

Figura 3. Evolución de la tasa de evaporación de la cobertura EVAPO-CONTROLG4 durante el ensayo.

Respecto a los efectos de ambas coberturas sobre la calidad del agua, los resultados ponen de manifiesto que no se ha producido una estratificación térmica en ninguna de las balsa. Por lo tanto, un valor medio de cada parámetro para el perfil de profundidad representa de forma adecuada el comportamiento del agua frente a la instalación de la cobertura flotante modular

La conductividad eléctrica se ha mantenido prácticamente constante a lo largo de los ensayo, ya que la cobertura permite recuperar la práctica totalidad de la precipitación, compensando las perdidas por evaporación y su posible efecto en la salinidad. La concentración de clorofila-a y el valor de turbidez también ha sido prácticamente nulos 
durante el ensayo experimental, evidenciando la eficiencia de la cobertura como técnica de control de la concentración de microalgas en la balsa de riego. Las Figs. 4 y 5 muestran la evolución de los parámetros de calidad temperatura del agua $\left(T_{a}\right)$, conductividad eléctrica $(C E)$, clorofila - a $(C l-a)$, turbidez $\left(T_{r}\right)$ y oxígeno disuelto $(O D)$ como valor medio de los registrados a 6 profundidades del perfil de agua para las coberturas EVAPO-CONTROL-2 y EVAPO-CONTROL-G4 respectivamente.

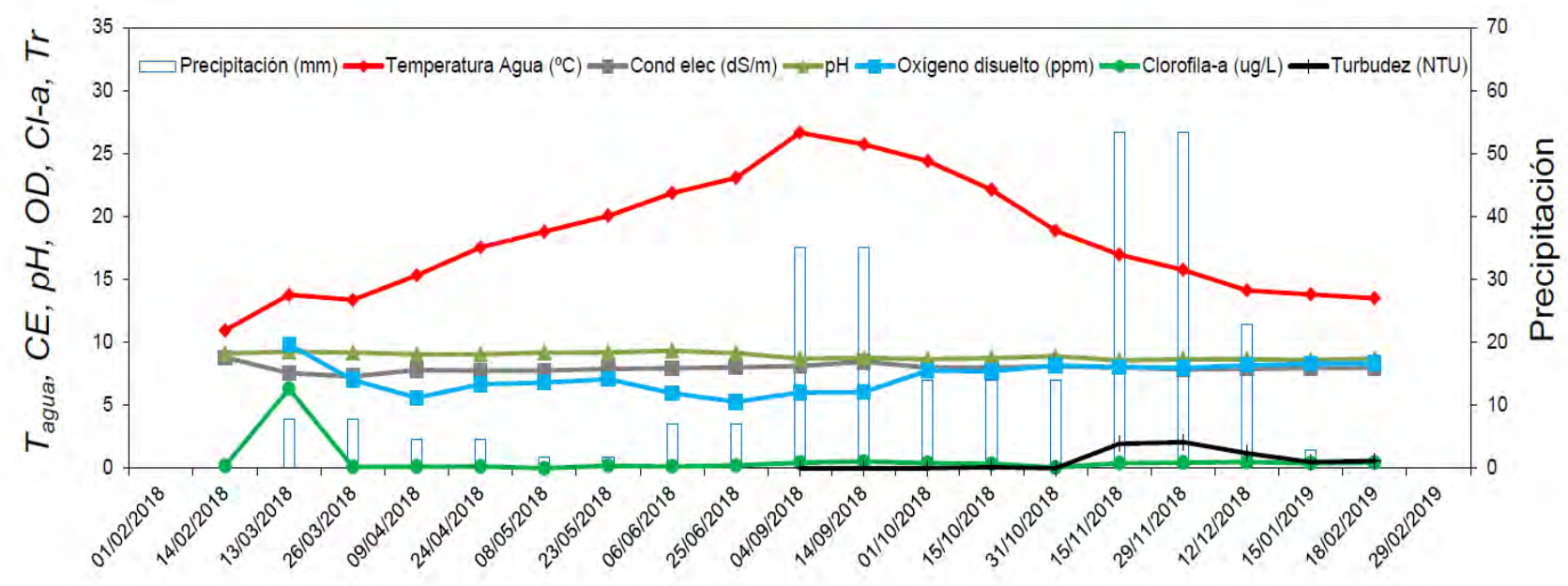

Figura 4. Evolución de los parámetros de calidad de agua analizados durante el ensayo experimental de la cobertura EVAPO-CONTROL-2.

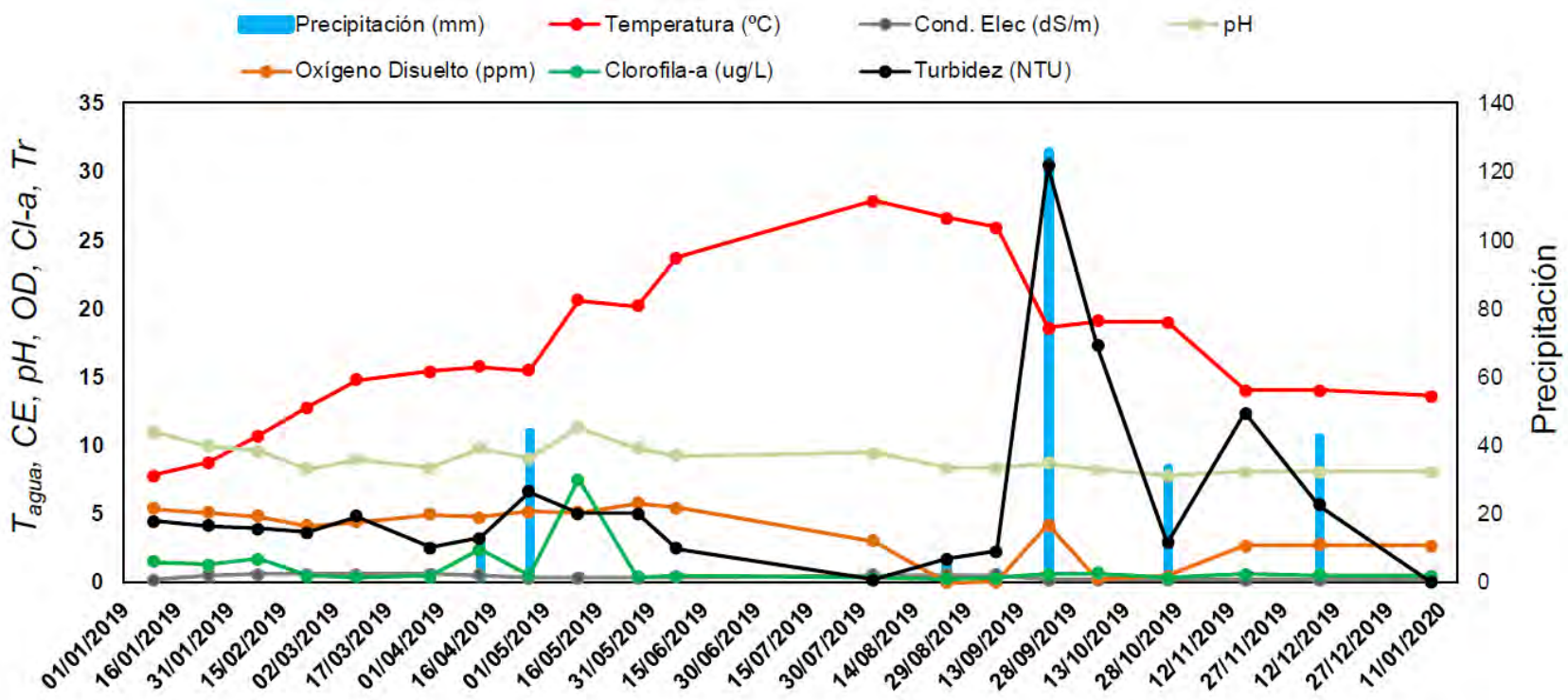

Figura 5. Evolución de los parámetros de calidad de agua analizados durante el ensayo experimental de la cobertura EVAPO-CONTROL-G4.

\section{5- Conclusiones}

Este informe presenta los resultados obtenidos tras el ensayo experimental de dos prototipos de cobertura flotante modular, denominados EVAPO-CONTROL-2 y EVAPOCONTROL-G4. Los prototipos fueron evaluados durante sendos años en balsas agrícolas del sureste español con el fin de (1) determinar experimentalmente su eficiencia como tecnología para la reducción de la evaporación, y (2) verificar experimentalmente su efecto sobre la calidad de agua almacenada para su aplicación en riego agrícola. 
Los ensayos se han realizado en balsas de riego representativas de las condiciones existentes en las explotaciones agrarias del sureste español, y bajo las condiciones climáticas también representativas de esta región. Por tanto, los resultados de este trabajo resultan directamente extrapolables a balsas de riego impermeabilizadas con geomembranas para localizaciones del sureste español y, de forma más genéricas, a balsas de riego impermeabilizadas en el ámbito mediterráneo.

La cobertura ha presentado el comportamiento mecánico esperado, sin que se haya observado durante el ensayo de la cobertura EVAPO-CONTROL-G4 el apilamiento de módulos como consecuencia de la fuerza de arrastre del viento. Los módulos han permanecido en todo momento sobre la superficie de agua en la balsa, cubriendo la totalidad de la superficie.

La cobertura EVAPO-CONTROL-G4 ha conseguido reducir la evaporación en un $77,52 \%$ durante el ensayo experimental de un año, mejorando ligeramente los resultados del prototipo anterior EVAPO-CONTROL-2, que redujo la evaporación en un $76,16 \%$ al ser evaluado durante un año en una balsa experimental localizada en el Campo de Cartagena. Por tanto, este ensayo confirma que se trata de una tecnología eficiente para la reducción de la evaporación en balsas de riego, y que el nuevo prototipo mejora el comportamiento de la versión anterior. El factor de reducción de la evaporación no ha sido constante a lo largo del año, sino que ha variado desde el $70,80 \%$ en el mes de enero hasta el $81,56 \%$ en el mes de agosto.

Respecto a los efectos de las coberturas EVAPO-CONTROL-2 y EVAPO-CONTROL G4 sobre la calidad del agua, la conductividad eléctrica se ha mantenido en valores muy reducidos y condicionados a la entrada de agua directa de lluvia. La concentración de clorofila-a (proxy de presencia de algas en la balsa) y el valor de turbidez también ha sido prácticamente nulos tras la instalación de la cobertura flotante, evidenciando la eficiencia de la cobertura como técnica de control de la concentración de microalgas en la balsa de riego.

Como consecuencia de estos resultados experimentales, se puede afirmar que la cobertura EVAPO-CONTROL G4 ha alcanzado unas especificaciones técnicas que la hacen muy adecuada para el control de la evaporación y mantenimiento de la calidad del agua en balsas de riego de tamaño moderado, presentando otras ventajas como la sencillez en su instalación y la ausencia de mantenimiento y riesgos climáticos.

\section{6- Agradecimientos}

En contenido de esta comunicación es fruto de las actividades desarrolladas en el marco de los contratos de investigación y desarrollo "Evaluación experimental del sistema de cobertura flotante modular EVAPO-CONTROL-2" y "Evaluación experimental del sistema de cobertura flotante modular EVAPO-CONTROL G4", desarrollados durante los años 2018 y 2019 respectivamente por la Universidad Universidad Politécnica de Cartagena y la empresa Arana Water Management s.I.

\section{7- Referencias}

Allen, R.G., Pereira, L.S., Raes, D., Smith, M., 1998. Crop evapotranspiration. guidelines for computing crop water requirements. Irrigation and Drainage Paper 56, FAO, Rome, 300 pp. 
Brown, J.A. 1988. The potential for reducing open water evaporation losses: a review. Hydrology and Water Resources Symposium 1988. Camberra, Australia. pp 108-115.

Brutsaert, W., 1982. Evaporation into the Atmosphere. Reidel, Dordrecht, 299 pp.

Craig, I., Green, A., Scobie, M., Schmidt, E., 2005. Controlling Evaporation Loss from Water Storages. NCEA Publication No 1000580/1, Queensland (Australia).

Gallego-Elvira, B., Finch, J.W., Baille, A., Martinez-Álvarez, V., 2009. Evaporation from onfarm storages under a semiarid climate: Measurement and modelling. International Workshop on Evaporation from Reservoirs. Gold Coast, Australia.

Harbeck, G.E., 1962. A practical field technique for measuring reservoir evaporation utilizing mass-transfer theory. U.S. Geol. Surv. Prof. Paper. 272-E: 101-105.

Maestre Valero, J.F., Martínez-Granados, D., Martínez-Alvarez, V., Calatrava, J., 2013. Socio-Economic Impact of Evaporation Losses from Reservoirs Under Past, Current and Future Water Availability Scenarios in the Semi-Arid Segura Basin. Water Resour Manage. 27:1411-1426.

Martínez, V., Baille, A., Molina, J.M., González-Real, M.M., 2006. Efficiency of shading materials in reducing evaporation from free water surfaces. Agricultural Water Management, 84: 229-239.

Martínez Alvarez, V., González-Real, M.M., Baille, A., Molina-Martínez, J.M., 2007. A Novel Approach for Estimating the Pan Coefficient of Irrigation Water Reservoirs. Application to South Eastern Spain. Agricultural Water Management, 92:29-40.

Martínez Alvarez, V., Calatrava Leyva, J., Maestre Valero, J.F., Martín Górriz, B., 2009. Economic assessment of shade-cloth covers for agricultural irrigation reservoirs in a semiarid climate. Agricultural Water Management 96:1351-1359.

Molina Martínez, J.M., 2004. Caracterización y modelización de la evaporación en tanques evaporímetros y embalses de riego. Evaluación de los efectos de mallas de sombreo. PhD Thesis, Agricultural and Food Department, Polythecnic University of Cartagena, Spain, 207pp. 22 